\title{
Kosten und Nutzen in der Krankenversorgung: Auf der Suche nach der richtigen Bewertung
}

Noch nie dürften sich im deutschen Gesundheitswesen so viele Menschen Gedanken über die Methodik einer angemessenen Kosten-Nutzen-Bewertung gemacht haben wie in den Jahren 2007 und 2008. Ausschlaggebend dafür waren das zum 1.4.2007 in Kraft getretene GKV (Gesetzliche Krankenversicherungen)-Wettbewerbsstärkungsgesetz und die zum 24.1.2008 erschienene erste Version des IQWiG-Methodenpapiers für die „Bewertung von Verhältnissen zwischen Nutzen und Kosten im System der deutschen gesetzlichen Krankenversicherung“. Belege dafür sind der Jahresbericht 2007 des IQWiG (Institut für Qualität und Wirtschaftlichkeit im Gesundheitswesen), bei dessen Lektüre man den Eindruck gewinnt, dass das Institut an die Grenze seiner Leistungsfähigkeit gestoßen ist, und die offiziellen 46 Stellungnahmen von Herstellern, Institutionen, Fachgesellschaften und anderen Organisationen, die beim IQWiG zu seinem Methodenpapier für die Kosten-NutzenBewertungen eingegangen sind; ganz zu schweigen von der Vielzahl von Tagungen, Publikationen, Gutachten und Pressemitteilungen. Der Ressourcen- und Emotionenverbrauch innerhalb und noch viel mehr außerhalb des IQWiG war jedenfalls volkswirtschaftlich nicht unerheblich.

Streng genommen ging es vielen ja auch nicht um die Methodik einer allgemein einsetzbaren Kosten-Nutzen-Bewertung, sondern um das vom IQWiG vorgeschlagene Verfahren zur Festlegung von Höchstbeträgen für innovative diagnostische und therapeutische Leistungen. Wegen der Fokussierung auf die Höchstpreis-Diskussion verlor man jedoch die Hausaufgaben bei den Schritten, die einer Höchstpreis-Festlegung vorausgehen, aus den Augen: das Erstellen von Methoden und Kriterien für die Nutzen-Bewertung und für die allgemeine Kosten-Nutzen-Bewertung, für die zudem laut §35b Abs. 1 SGB V das IQWiG eine „hohe Verfahrenstransparenz“ zu gewährleisten hat und darüber hinaus „eine angemessene Beteiligung“ u.a. von Sachverständigen der medizinischen und pharmazeutischen Wissenschaft und Praxis, den Arzneimittelherstellern und den Patientenvertretern. Trotz der mittlerweile zum 27.5.2008 erschienenen Version 3.0 des Allgemeinen Methodenpapiers und der Version 1.1 des Kosten-Nutzen-Bewertung-Papiers vom 14.10.2008 sind noch längst nicht alle Probleme gelöst.
Daher ist die Veröffentlichung des vom Verband Forschender Arzneimittelhersteller (VFA) in Auftrag gegebenen Gutachtens in einem Supplement, das dieser DMW Ausgabe beiliegt, nachdrücklich zu begrüßen. Es enthält konkrete Vorschläge zu den Verfahrensweisen und Methoden der Nutzen-Bewertung.

\section{kurzgefasst}

Bevor man sich über die Methoden der Bestimmung von Höchstpreisen für innovative Behandlungen Gedanken macht, sollten die Probleme der Nutzenbewertung und der Kosten-Nutzen-Bewertung gelöst sein.

\section{Abhängigkeit und Unabhängigkeit von Gutachtern und Stellungnehmern}

Die Suche nach unabhängigen Fachexperten gleicht der Suche nach Phantomen. Eine absolute Unabhängigkeit von Fachexperten gibt es nicht. Es gibt nur graduelle Abhängigkeiten. Selbst jene Wissenschaftler, die ihre Projekte mit eigenen Mitteln finanzieren oder „neutrale“ Finanzierungsquellen in Anspruch nehmen, zeigen sich oft abhängig von ihren eigenen Forschungsergebnissen. Und natürlich ist auch der Schreiber dieser Zeilen - stolz auf seine gefühlte Unabhängigkeit - von seiner wissenschaftlichen Sozialisierung abhängig.

Wegen der ubiquitär vorhandenen Abhängigkeiten waren die Hoffnungen auf die Fähigkeiten klinischer Studien und systematischer Reviews groß: Sie sollten unabhängige Belege für die Wahrheit liefern. Doch die Erfahrung lehrte: Es gibt keine perfekten klinischen Studien. Sogar die zu Recht gepriesene randomisierte Studie hat einen wesentlichen Nachteil, der sie vom Versorgungsalltag unterscheidet: Man muss die Patienten bitten, einer zufälligen Zuteilung ihrer Behandlung zuzustimmen.

Die Erwartungen an die Evidenz-basierte Medizin (EbM), nämlich den existierenden klinischen Studien mit aller Neutralität mittels eines stringenten Regelwerks Empfehlungen für die Behandlung einzelner Patienten abringen zu können, haben sich insofern nicht erfüllt, als dass Ärzte und Patienten ihre Entscheidungen nicht an die Ergebnisse einer Evidenzbasierung delegieren können, sondern immer noch selbst entscheiden müssen, jetzt allerdings auf einem höheren Wissensniveau. Das gleiche gilt natürlich

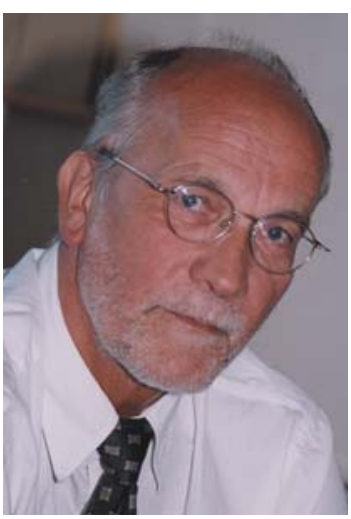

H.-K. Selbmann

Gesundheitsökonomie

Schlüsselwörter

Nutzen-Bewertung

IQWiG

Evidenz-basierte Medizin NICE

Keywords

Assessment of benefit - IQWiG

Evidence-based medicine

NICE

Siehe auch Supplement Nr. 7 zur DMW Nr. 49 (5.12.2008): Verfahrensweisen und Methoden zur NutzenBewertung von Arzneimitteln in Deutschland -

Ein Auftragsgutachten

Institut

Universität Tübingen

eingereicht 2.11.2008 akzeptiert $\quad 6.11 .2008$

Bibliografie

Dol 10.1055/s-0028-1100965 Dtsch Med Wochenschr 2008 ; 133: 2579-2581 • (c) Georg Thieme Verlag KG Stuttgart . New York · ISSN 0012-0472

Korrespondenz

Prof. Dr. H. K. Selbmann Institut für Medizinische Informationsverarbeitung Büro Rangendingen Panoramastr. 22

72414 Rangendingen 
auf der Makro-Ebene auch für die Entscheidungen des Gemeinsamen Bundesausschusses (G-BA). Er muss u.a. zwischen Nutzen und Schaden, zwischen individuellem und sozialem Nutzen und zwischen verschiedenen gesellschaftlichen Wertevorstellungen abwägen, und dies alles vor dem Hintergrund einer mal mehr, mal weniger unsicheren Datenlage.

Der Mangel an Fachexperten, die weitestgehend unabhängig sind, hat übrigens die Arbeitsgemeinschaft für Wissenschaftliche Medizinische Fachgesellschaften (AWMF) schon gegen Ende der 90er Jahre bewogen, bei der Entwicklung von Leitlinien nicht ausschließlich auf die Evidenzbasierung zu setzen, sondern ihr strukturierte Konsensfindungstechniken anzuschließen. Diese Konsensfindungstechniken sind so angelegt, dass sie die Gefahr der Einflussnahme durch einzelne Abhängigkeiten reduzieren.

Die beiden internationalen Autoren des Gutachtens - Trudy Bekkering und Jos Kleijnen - sind bezüglich einer direkten Abhängigkeit unverdächtig, da sie sowohl für die Pharma- und MedizinprodukteIndustrie arbeiteten als auch u.a. vom NICE (National Institute for Health and Clinical Excellence, zuständig für England und Wales) und dem IQWiG als Fachexperten engagiert werden. Doch auch bei ihnen gibt es implizite Abhängigkeiten, bedenkt man z.B., dass sie aus unterschiedlichen Kommunikationskulturen und Gesundheitswesen kommen.

\section{kurzgefasst}

Es gibt keine absolute Unabhängigkeit unter den ausgewiesenen Fachexperten, sondern nur graduelle Abhängigkeiten. Auch die Evidenz-basierte Medizin enthält eine Vielzahl von Wertungen, bei denen Abhängigkeiten Einfluss nehmen können.

\section{Der Prozess der Berichterstellung des IQWiG}

Auch wenn die Autoren feststellen, dass ihr Gutachten nicht spezifisch für das IQWiG, sondern für alle Institute gilt, die vom G-BA möglicherweise mit Aufträgen bedacht werden, basieren ihre Optimierungsvorschläge im Wesentlichen doch auf dem Berichterstellungsverfahren des IQWiG. Seit 2007 sieht dieses Verfahren nach seiner Beauftragung des
Instituts durch G-BA oder Bundesministerium für Gesundheit (BMG) die Bildung einer institutsinternen Projektgruppe vor, die die wissenschaftliche Fragestellung in Abstimmung mit den Gremien des Auftraggebers und ggf. unter Einbeziehung öffentlich nicht bekannter, externer Fachexperten oder Meinungen einzelner Betroffener sowie, bei der Definition der patientenrelevanten Endpunkte, entsprechender Patientenorganisationen formuliert. Anschließend wird der Berichtsplan erstellt, zu dem die im Gesetz vorgegebene Öffentlichkeit innerhalb von 4 Wochen offiziell Stellung nehmen kann. Eine zweite Gelegenheit zur Stellungnahme - wiederum innerhalb von 4 Wochen nach Erscheinen - ergibt sich nach der Vorlage des Vorberichts, der auch den ggf. geänderten Berichtsplan enthält.

Nach Eingang der Stellungnahmen und ggf. daraus folgenden Modifikationen des Vorberichts wird vom IQWiG der Abschlussbericht für den Auftraggeber erstellt. Dieser enthält in der Regel auch Hinweise auf alle zum Vorbericht eingereichten Stellungnahmen. Nicht explizit zum IQWiG-Berichtserstellungsverfahren gehört, dass natürlich jeder nach der Fertigstellung des Abschlussberichts eine weitere Stellungnahme verfassen und direkt dem G-BA zuleiten kann.

\section{kurzgefasst \\ Das Berichtserstellungsverfahren des IQWiG sieht zwei Zeitpunkte für Stellungnahmen mit 4 Wochen Frist vor: jeweils nach der Publikation des vorläufigen Berichtsplans und des Vorberichts. Die vom IQWiG einge- bundenen Sachverständigen aus Wissenschaft und Praxis werden nicht bekannt gegeben.}

\section{Die wichtigsten Vorschläge des Gutachtens}

Das Grundverständnis des Qualitätsmanagements - es gibt nichts, was man nicht noch besser machen kann - gilt auch für einen so schwierigen Produktionsprozess wie den der Berichterstellung des IQWiG. Das Hauptverbesserungspotenzial sehen die Autoren des Gutachtens in der Herstellung von Transparenz und der Beteiligung der im Gesetz angesprochenen Gruppierungen und dies insbesondere in der Phase bis zur Erstel- lung des Vorberichts. Fragen wie „Wurden die verschiedenen, oft gegenläufigen Interessen bei der Feinformulierung der Fragestellung berücksichtigt und wer wurde konkret daran beteiligt?" oder „Wie sahen die Arbeitsergebnisse der ausgewiesenen Fachexperten aus, bevor sie vom IQWiG weiterverarbeitet wurden?" bleiben in der Tat beim IQWiGVerfahren derzeit im Dunkeln.

In Anlehnung an die Berichterstellung beim NICE schlagen die Autoren vor,

1. einen Scoping-Prozess bei der Entwicklung der Fragestellung einzuführen,

2. die Arbeit der externen Sachverständigen, die die Evidenzbewertung vornehmen, von der des Instituts, das die Empfehlungen für die Auftraggeber formuliert, zu trennen und

3. ein offenes Peer-Review-Verfahren durch Veröffentlichung der Stellungnahmen mit namentlicher Nennung der Reviewer durchzuführen.

Diesbezüglich hilfreich und lesenswert ist ihr detaillierter Vergleich zwischen den vom NICE und vom IQWiG angewandten Verfahren.

Die Idee, einen Scoping-Workshop durchzuführen, bevor die Arbeit an der Berichterstellung beginnt, ist in Deutschland nicht ganz neu. Er wurde u.a. auch von der AWMF als Sichtungsworkshop vorgeschlagen, um die einzubeziehenden Gruppierungen, die Patienten-Zielpopulation, die zu vergleichenden Interventionen, die patientenrelevanten Endpunkte und die Suche nach der besten, verfügbaren Evidenz so klar wie möglich $\mathrm{zu}$ beschreiben (www.uni-duesseldorf.de/AWMF/pdf/scoping.pdf). Wichtig erscheint dabei insbesondere, dass ein solcher Workshop neutral moderiert wird, denn das Aufeinandertreffen unterschiedlicher Motive und Wertvorstellungen lässt sich nicht immer vermeiden. Es wird die Aufgabe des G-BA sein - wenn er die Folgen seiner gesetzlich verursachten Neustrukturierung bewältigt hat beim Etablieren eines solchen, von vielen Seiten geforderten Scoping-Workshops den ersten Schritt zu machen.

National und international üblich ist mittlerweile, zwischen der internen und der externen Validität von Studien und systematischen Reviews zu unterscheiden. Die interne Validität meint die Vollzähligkeit 
der einzubeziehenden Studien, ihre Aussagensicherheit und die konsistente Schätzung der Effektstärken. Dazu gibt es inzwischen Seminare, auf denen man die entsprechenden Techniken lernen kann. Für die Feststellung der externen Validität - der Relevanz und der Anwendbarkeit im deutschen Versorgungsalltag bedarf es einer klinischen Bewertung der Studienergebnisse (oft auch clinical judgement genannt) durch ausgewiesene wissenschaftlich medizinische Fachexperten. Die Ableitung von Empfehlungen für den Versorgungsalltag lässt sich weniger gut standardisieren und führt wegen seiner größeren Ermessensspielräume auch zu einer größeren Variabilität der abgeleiteten Empfehlungen. Methodisch ist diese Variabilität u.a. darin begründet, dass die dabei auftretenden Fragen z.B. nach der Vereinigung von Nutzen und Nebenwirkungen zu einem Nettonutzen (in der Version 3.0 des Allgemeinen Methodenpapiers des IQWiG nur vage angesprochen), nach der Übertragbarkeit der Ergebnisse von Studienpopulationen auf die Zielpopulation oder nach der Importierbarkeit von ausländischen Studienergebnissen in das deutsche Gesundheitswesen mit Hilfe von Modellvorstellungen, Analogschlüssen und plausiblen Annahmen beantwortet werden müssen. Je größer die Variabilität ist, desto größer ist der Bedarf an Transparenz.

Zur Steigerung der Transparenz schlagen die Autoren des Gutachtens vor, dass die Arbeitsergebnisse der externen Fachexperten als eigenständige Leistung bekannt gemacht werden sollen. So könnte gewährleistet werden, dass ein eventueller Dissens zwischen den ausgewiesenen Fachexperten und dem IQWiG hinsichtlich der Schlussfolgerungen aus der Evidenzbasierung transparent und nachvollziehbar ist. Dieser Vorschlag ist aus wissenschaftlich-medizinischer Sicht ebenfalls zu begrüßen, auch wenn dazu das Verfahren noch detailliert geplant werden müsste. Bisher waren die Fachexperten als anonyme „Zuarbeiter“ für das IQWiG eingebunden und mussten sich mit dem IQWiG-Vorbericht identifizieren, auch wenn dieser nicht ihren Vorstellungen entsprach. Die Anonymitätsgarantie war vom IQWiG als Schutz für die Fachexperten gedacht. Ausgewiesene Fachexperten sind aber deshalb ausgewiesen, weil sie eben in der Vergangenheit ihre Arbeitsergebnisse nicht unter den Scheffel gestellt haben. An der Zahl und der Qualität der eigenständigen Produkte wurde ihre Leistungsfähigkeit gemessen, die ihrerseits dann Einfluss auf die leistungsbezogene Mittelverteilung ihrer Arbeitgeber hatte.

Die dritte Vorschlag der Autoren, der nach einem offenen Peer-Reviewing mit voller Namensnennung, ist noch am einfachsten zu realisieren und wurde vom IQWiG jetzt bei der Entwicklung der Methodik zur Kosten-Nutzen-Bewertung auch schon praktiziert. Dort sind alle 46 Stellungnahmen im Internet mit einer zusammenfassenden Würdigung seitens des IQWiG publiziert. Das Problem liegt weniger in der Veröffentlichung der Stellungnahmen oder der Vermittlung ihrer Verwendung - letzteres ließe sich durchaus noch optimieren - als im Verfassen der Stellungnahmen selbst. Für viele Fachgesellschaften und Organisationen kann die dafür anberaumte Vier-Wochen-Frist zu Kapazitätsproblemen führen. Da andererseits nachzuvollziehen ist, dass eine reine Verlängerung der Stellungnahmefrist die Berichtsabgabe an den Auftraggeber verzögern würde, sind die Fachgesellschaften und Organisationen gehalten, das Verfassen der Stellungnahmen noch stärker zu professionalisieren. Dass dies allerdings mit einem zusätzlichen Ressourcenverbrauch einhergeht und $\mathrm{zu}$ bisher noch ungelösten Finanzierungsproblemen führt, ist vorauszusehen.

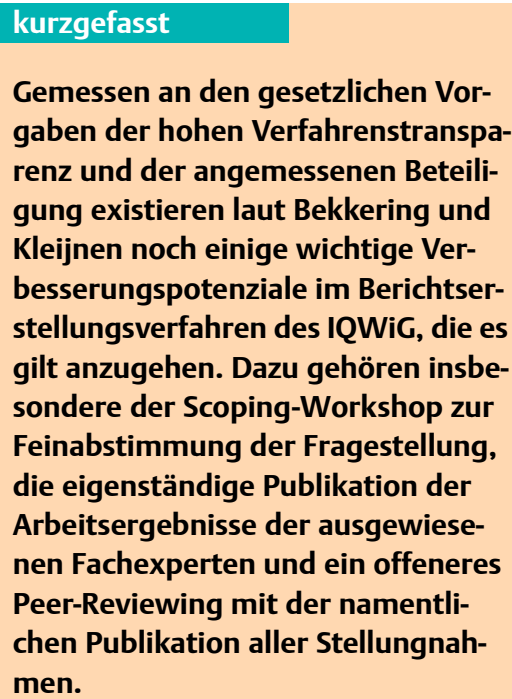

\section{Empfehlungen}

$\nabla$

Das Gutachten ist eine gute Vorarbeit, um sich auf die Suche nach Verbesserungspotenzialen im IQWiG-Berichtsver- fahren zu machen. Es vermittelt viel Grundwissen über international diskutierte und geübte Verfahrensweisen und erleichtert die Diskussionen über Verfahrensoptimierungen.

Leider befasst sich das Gutachten nur sehr kurz mit den Unterschieden zwischen einer isolierten Nutzen-Bewertung und einer Nutzen-Bewertung im Rahmen einer Kosten-Nutzen-Bewertung. Nach den Plänen des IQWiG ist bei KostenNutzen-Bewertungen ein zweistufiges Vorgehen vorgesehen: Nur beim Vorliegen von konkreten Belegen für einen Nutzenzugewinn werden die NutzenKosten-Verhältnisse bewertet.

Abgesehen davon, dass durch das zeitlich gestaffelte Vorgehen viel Zeit verloren geht, kann dieses zweistufige Vorgehen nur dann zu konsistenten Empfehlungsvorschlägen führen, wenn in beiden Stufen dieselben Bewertungsmethoden und -daten für den Netto-Nutzen-Zugewinn (z.B. gleiche Nutzendimensionen, gleiche Nutzen-Schaden-Abwägungen, gleiche Perspektiven und gleiche Zeiträume) verwendet werden. Zur Untersuchung des Zusammenspiels zwischen isolierter Nutzen-Bewertung und nachfolgender Kosten-Nutzen-Bewertung wäre ein zweites Gutachten lohnenswert.

Autorenerklärung: Der Autor hat über 30 Jahre u.a. Medizinische Statistik, Epidemiologie und Gesundheitsökonomie gelehrt. Er ist Mitglied des Präsidiums der AWMF (Arbeitsgemeinschaft der Wissenschaftlichen medizinischen Fachgesellschaften) und dort zuständig für die Leitlinienentwicklung. 